\title{
Accompagnement biographique et construction du comportement alimentaire chez les enfants avec un trouble du spectre de l'autisme
}

\section{Anne-Claude Luisier}

Les enfants et les adolescents avec un trouble du spectre de l'autisme (TSA) rencontrent fréquemment des problèmes alimentaires, en particulier une grande sélectivité qui rend leur vie et celle de leurs proches compliquées. Cette recherche qualitative utilise une approche biographique pour une étude de cas auprès d'une adolescente avec un TSA qui présente une forte sélectivité alimentaire. Dans un premier temps, après une brève présentation du TSA, les enjeux sous-jacents à l'acte de manger sont décrits. Puis, des éléments de compréhension du lien que l'adolescente a construit avec le monde alimentaire sont proposés sur la base de son histoire de vie.

\section{Introduction}

Dans cette étude, nous évaluons l'intérêt des approches qualitatives biographiques lors d'interventions d'éducation à l'alimentation auprès d'enfants avec un trouble du spectre de l'autisme (TSA). Cette étude a été menée dans le cadre d'une thèse de doctorat et du CAS (Certificate of Advanced Studies) en recueil de récit de vie proposé par le département de formation continue de l'Université de Fribourg. Nous avons recueilli le récit de vie, selon le cadre théorique et épistémologique des histoires de vie (Lainé, 2004; Pineau \& Legrand, 2013), de Lamia ${ }^{1}$ une adolescente de 17 ans avec un TSA qui rencontre de grandes difficultés alimentaires. Nous avons mené conjointement des recherches quantitatives et hypothético-déductives sur la construction du comportement alimentaire chez des enfants et des adolescents ${ }^{2}$ avec un TSA (Luisier, 2017).

Proposer une éducation à l'alimentation demande de tenir compte de la construction complexe du comportement alimentaire. Si cette construction est très étudiée chez l'enfant au développement typique (DT), elle l'est beaucoup moins chez l'enfant avec un TSA. Pour le professionnel, il est important d'en comprendre les spécificités pour pouvoir ajuster sa posture de façon adéquate lors de l'accompagnement d'un enfant avec un TSA que ce soit lors de l'inclusion en milieu ordinaire 3 ou dans une institution. 
L'éducation à l'alimentation est un défi particulier auprès des enfants avec un TSA. En effet, si des problèmes alimentaires ${ }^{4}$ sont rencontrés chez 13 à $50 \%$ des enfants au développement typique (DT), ils semblent présents chez plus de $80 \%$ voire $90 \%$ des enfants avec un TSA (Cermak, Curtin \& Bandini, 2010; Fodstad \& Matson, 2008; Herndon, DiGuiseppi, Johnson, Leiferman \& Reynolds, 2009; Johnson, Foldes, Demand \& Brooks, 2015; Matson, Fodstad \& Dempsey, 2009; Nadon, Feldman, Dunn \& Gisel, 2011; Nadon, Feldman \& Gisel, 2013). La difficulté la plus fréquemment décrite est la sélectivité alimentaire $^{5}$ avec comme conséquence un répertoire alimentaire restreint qui prédispose l'enfant avec un TSA à un risque de déficit nutritionnel (Ledford \& Gast, 2006; Marshall, Hill, Ziviani \& Dodrill, 2014; Sharp, Jaquess \& Lukens, 2013; Zimmer et al., 2012).

Ces difficultés alimentaires fréquentes péjorent la qualité de vie de l'enfant ainsi que celle de sa famille (Marshall et al., 2014; Schaaf, Toth-Cohen, Johnson, Outten \& Benevides, 2011). Elles ont aussi un impact sur le développement de son réseau social. Des activités telles que fêtes d'anniversaire, repas au restaurant, fêtes de famille deviennent difficiles (Nadon, Ehremann Feldman \& Gisel, 2008). Les repas quotidiens sont source de stress pour les parents (Nadon et al., 2011). Ces attitudes contribuent à l'isolement social de l'enfant et de sa famille et renforcent la perception de la différence.

Des études quantitatives (Luisier, 2017; Luisier et al., 2015, 2017) nous ont permis de mettre en évidence des corrélations entre la perception visuelle et olfactive des enfants avec un TSA et leur difficulté à accepter de nouveaux aliments, ce qui n'est pas le cas pour les enfants au DT. Ces travaux ne permettent cependant pas de saisir le rapport subjectif aux aliments qu'entretiennent les personnes avec un TSA. C'est ce rapport que nous souhaitions mieux comprendre grâce à l'étude biographique que nous avons menée avec Lamia.

Dans un premier temps, après une brève présentation du TSA, nous décrirons les enjeux sous-jacents à l'acte de manger. Nous définirons ensuite la méthodologie mise en ouvre pour recueillir et analyser le récit de Lamia. Nous apporterons finalement des éléments de compréhension du lien qu'elle a construit avec le monde alimentaire.

\section{Le trouble du spectre de l'autisme}

Le DSM-5 (APA, 2015) classe le TSA dans les troubles neuro-développementaux. Il définit comme critères diagnostiques (1) un déficit persistant de la communication et des interactions sociales, comme la difficulté à soutenir une conversation bidirectionnelle normale ou un manque d'intérêt pour les pairs et (2) le caractère restreint et répétitif de comportements, d'intérêts ou d'activités comme l'intérêt excessif pour certains objets, l'intolérance au changement ou une réactivité inhabituelle à certains stimuli sensoriels. 
Donnellan, Hillset et Leary (2013) rappellent que les bases neurologiques propres aux troubles autistiques conditionnent le développement et le fonctionnement de la personne, en particulier par une façon différente d'organiser et de réguler les informations sensorielles et motrices. Or, ces informations sont à la base de la construction du comportement alimentaire. Selon de Suremain et Razy (2012),

le passage de l'enfance à l'âge adulte est jalonné d'étapes physiologiques auxquelles correspondent divers apprentissages parmi lesquels celui, essentiel, des pratiques alimentaires. Éminemment variables, elles se fondent sur des représentations de l'alimentation, mais également de la "personne» et de son corps - constituée d'entités, de composantes, d'organes et de substances -, et de son développement psychomoteur. (p. 455)

\section{L'acte de manger}

Pour comprendre le rapport qu'entretient un mangeur avec son alimentation, il faut considérer plusieurs dimensions. En effet, manger est un acte complexe. Etiévant et al. (2010), précisent que

l'alimentation ne se réduit pas à un ensemble de nutriments, elle met en jeu des associations d'aliments; support de représentations mentales et culturelles, elle fait partie d'un ensemble de comportements individuels et collectifs et s'inscrit dans un contexte d'évolutions démographiques et de modifications des modes de vie (p. 3).

Les dimensions de l'alimentation peuvent être regroupées en trois fonctions principales (Agence nationale de sécurité sanitaire de l'alimentation, de l'environnement et du tavail [ANSES], 2010; Lecerf, 2013):

1. Nourrir - couvrir les besoins énergétiques et nutritionnels, de façon à garder l'individu en vie et à assurer le bon fonctionnement biologique. Manger est un acte vital, obligatoire. C'est peut-être l'un des actes les plus profondément autonomes de l'être humain. Le refus catégorique de manger rencontré à tout âge de la vie et qui peut conduire à la mort est parfois un sursaut d'autonomie, une façon ultime de se revendiquer sujet quand tout semble échapper à son contrôle, quand la dépendance aux autres est ressentie comme insupportable. Et ce refus existe encore plus fortement lorsqu'il rend l'autre ou les autres impuissants.

2. Réunir - relier avec les autres humains (famille, société, culture...). L'acte de manger pourrait être considéré comme la fonction sociale première. En effet, depuis les premières sociétés humaines, au temps des chasseurs cueilleurs, la société organise sa vie autour de l'approvisionnement alimentaire (Fischler, 2012). Au niveau individuel, dès ses premières heures, le nourrisson communique ses préférences alimentaires par ses mimiques à son entourage (Chiva, 
1979, 1985). Ensuite, au hasard des repas et des convives rencontrés, l'enfant s'ouvre à d'autres, différents de lui, pour construire une compréhension du monde, de l'autre et de lui-même.

3. Réjouir - contribuer à l'équilibre psychologique individuel par l'intermédiaire des affects et des émotions. Manger est tout d'abord un acte sensoriel. Les messages reçus par les sens et l'interprétation qui en découle vont conditionner les émotions ressenties pour l'aliment, l'envie ou non de l'ingérer. Ce qui est source de plaisir ou de dégoût dépend de toutes les expériences avec le monde alimentaire et est donc très individuel. Selon les aliments consommés, le plaisir ressenti et le sens donné, le mangeur expérimente, ou pas, la satisfaction de répondre à ses besoins tant physiologiques que psychologiques.

Nous allons dans cette recherche investiguer comment le recueil d'un récit de vie permet de mieux comprendre le comportement alimentaire individuel d'une adolescente avec un TSA.

\section{La méthodologie}

Nous avons opté pour le cadre méthodologique et épistémologique des histoires de vie (Lainé, 2004; Pineau \& Legrand, 2013) qui «oriente la recherche vers une mise en évidence du sens que revêt une expérience pour un acteur, par repérage et analyse des traces que cet auteur laisse, pour reconstituer des ensembles signifiants» (Vanini De Carlo, 2014, p. 3). Les approches biographiques permettent de récolter en profondeur le point de vue du sujet, de prendre en compte la subjectivité du chercheur afin de mieux comprendre, entre autres, ce qui vient du monde ordinaire et ce qui vient du monde de l'autisme, d'être source d'apprentissages et de transformation tant pour le chercheur que pour le sujet d'étude, d'adopter une perspective qui tienne compte du cours de la vie (life course) et des savoirs qui en émergent, savoirs utiles pour, par exemple, concevoir des actions d'éducation à la santé (Wethington, 2005).

Le chercheur recueille un récit et accompagne l'émergence d'une histoire sans savoir à l'avance quelle en sera l'intrigue. Le narrateur (sujet de recherche) et le narrataire (chercheur) sont co-auteurs del'histoire qui s'écrit. Cette co-construction de l'histoire a une portée émancipatoire ${ }^{6}$ pour le sujet (Lamia), ainsi cette méthodologie permet non seulement de recueillir des données de recherche mais aussi d'accompagner le sujet et d'initier des transformations ${ }^{7}$ chez lui.

Pour le chercheur, cela signifie d'envisager sa recherche comme une recherche à la "seconde personne» (Rommetveit, 2003). Le sujet d'étude n'est pas observé de l'extérieur, mais à l'intérieur de la relation qu'il crée avec le chercheur qui adopte une posture dialogique. La relation est possible, car le chercheur assume sa condition de sujet dans la relation et parle ainsi avec son sujet d'étude et non pour lui. La pensée dialogique est une "pensée qui aborde le monde, les événements, du point de vue de la relation à l'autre (Souriau, 2013)». 


\section{La rencontre avec Lamia}

À la demande d'une association de parents d'enfants avec un TSA, nous avions présenté notre recherche en cours sur l'alimentation des enfants avec un TSA et Lamia et sa maman s'étaient portées volontaires pour y participer.

Lamia a 15 ans au moment de cette prise de contact, elle en a 17 au moment du recueil de son récit. Elle a suivi dans son enfance un cursus scolaire ordinaire. Très bonne élève, très discrète en classe, elle se fait remarquer dans la cour de récréation par des réactions fortes envers ses camarades. Elle présente déjà une grande sélectivité alimentaire. Sa mère dit qu'elle a compris très vite qu'elle ne pourrait pas agir avec Lamia comme avec ses autres enfants. Ce n'est toutefois que lors du passage à l'école secondaire que la situation est devenue si difficile que Lamia, presque 13 ans, est hospitalisée et que le diagnostic de «syndrome d'Asperger» est posé ainsi que celui de "haut potentiel». Lamia a terminé son école obligatoire et essaie actuellement de construire un projet professionnel.

\section{Contractualisation}

Le recueil de récit de vie a été effectué dans le cadre d'une thèse de doctorat dont le protocole a été validé par la commission Cantonale Valaisanne d'Éthique Médicale (IRB number: CCVEM 022/14). Le contrat a été conçu avec Lamia et sa mère qui l'ont co-signé avec l'auteure de la recherche.

\section{Recueil du récit}

Le recueil s'est effectué sous la forme d'entretiens.

Deux formes/contextes ont été choisi(e)s pour les entretiens:

- l'entretien libre: durant six entretiens, Lamia a raconté librement sa vie. Elle a parfois choisi d'aborder les entretiens en apportant des objets, comme des éléments de ses collections ou des dessins qu'elle avait réalisés, elle a aussi eu recours à des photos stockées dans son téléphone portable;

- les dégustations ${ }^{8}$ : à deux reprises, des dégustations ont été partagées pour mieux comprendre comment Lamia aborde les situations alimentaires et sensorielles.

L'analyse - Reconfiguration du récit et interprétation L'analyse vise à mettre en évidence le sens que Lamia donne à son alimentation.

Dans un premier temps, nous avons reconfiguré la «modélisation narrative» (Vanini De Carlo, 2014a) ${ }^{9}$ sous la forme d'un récit phénoménologique (Paillé $\&$ Mucchielli, 2016, pp. 152-159) que nous appellerons aussi «histoire» 10. Autrement dit, nous avons extrait de la narration les moments durant lesquels Lamia parle de son alimentation, nous les avons regroupés en entités porteuses de sens (catégories) et nous les avons reconfigurés pour constituer une histoire. Nous avons choisi comme catégories pour organiser les phénomènes, les fonctions principales de l'alimentation évoquées ci-avant. Cette reconfiguration écrite a été relue par Lamia qui a validé tant le contenu que la forme. 
Dans un second temps, nous avons cherché à interpréter les phénomènes décrits par Lamia et à en comprendre la signification. Les liens de compréhension que nous proposons ainsi que leur formulation écrite ont tous été discutés avec Lamia. Elle a confirmé trouver son mode de fonctionnement dans l'interprétation que nous avons faite.

Si l'analyse permet de réécrire une histoire (qui donne une signification à un moment donné) à partir des propos du narrateur (participant de l'étude), elle n'est pas sans effet sur le chercheur qui porte un regard réflexif sur sa propre vision du monde, ses connaissances et sa pratique. "L'interprétation au sens herméneutique est à concevoir comme un moment où c'est aussi l'interprétant qui est 'créé: ce lecteur-analyseur produit en fait un nouveau texte, et devient d'une certaine manière co-auteur de l'histoire, ou mieux, d'une version de l'histoire» (Vanini De Carlo, 2014a, p. 6).

\section{Résultats et analyses ${ }^{11}$}

Nous avons ainsi distingué les deux temps de l'analyse en présentant en dans le texte mis en retrait des extraits du récit phénoménologique ${ }^{12}$ et suivi de l'interprétation.

Quand j'ai rencontré Lamia pour la première fois, il y a deux ans, lors d'une conférence que j'ai donnée sur le thème de l'alimentation, elle exprime son envie de me parler de son rapport au monde alimentaire. Elle m'avait dit simplement "ça va être difficile de trouver un apprentissage, car si je pars de la maison, je vais mourir.» Face à mon étonnement, elle avait précisé, «je vais mourir de faim. Je ne peux manger qu'à la maison, ce que cuisine ma maman.»

La narration de ce récit a lieu pour Lamia durant l'adolescence, moment de grands changements, moment charnière de la vie. Lamia évoque de façon détaillée et récurrente certaines thématiques, en particulier sa passion dévorante pour le monde des mangas ou pour ses collections. Ces thématiques occupent beaucoup de temps dans ses récits. Nous percevons ces intérêts envahissants comme des tentatives de ralentir le cours du récit, des "pics de ralentissement» 13 (Vanini De Carlo, 2014a), à l'image de ce qui se passe actuellement dans sa vie. En effet, elle semble s'être créé une sorte de no man's land depuis la fin de la scolarité obligatoire comme pour retarder le plus possible le début d'une vie d'adulte qui arrive trop vite pour elle. Elle n'est pas prête à s'investir dans un apprentissage ou des études. Elle se réfugie dans ses passions. Elle est consciente des exigences de la société à l'égard des jeunes de son âge même si elle voit bien qu'elle n'arrive pas à y répondre quand, par exemple, elle continue à collectionner des figurines enfantines ou quand elle craint les lieux où il $\mathrm{y}$ a beaucoup de monde. Entrer dans ce monde des adultes lui demanderait de quitter ses passions. Cette étape de vie se complique encore pour elle de par son TSA. Elle sait qu'elle est différente même 
si elle voudrait tellement que cela ne se voie pas. Alors que faire si ce n'est arrêter le temps? Elle est toutefois consciente que cet arrêt sur image ne sera pas éternel, mais elle a besoin justement de temps pour apprivoiser les changements. Du reste, en disant, par exemple, que les mangas sont aussi une occupation d'adultes ou qu'elle aimerait écrire un livre, elle se prépare à rendre le futur possible.

\section{Le monde alimentaire selon Lamia}

\section{Nourrir - Manger est un acte vital, un acte porteur d'autonomie}

Pour Lamia, l'alimentation est un combat dans lequel se mêlent à la fois les aspirations d'autonomie d'une adolescente ordinaire et celles plus sécuritaires d'une adolescente extra-ordinaire. Les difficultés de Lamia sont mises en exergue dans son rapport à l'alimentation. Elle recherche toujours les "mêmes goûts 14 ", c'est pourquoi elle dépend presque totalement de la cuisine de sa mère. Elle peut toutefois accepter cette dépendance, car elle a droit à un régime particulier qu'elle considère comme une "dominance» par rapport à sa mère. Nous y voyons une forme d'autonomie, car c'est elle qui décide.

Pour Lamia, l'alimentation a toujours été le signe visible d'une différence invisible. Lors des fêtes avec sa famille élargie, elle n'arrive pas à manger le menu proposé. Ce n'est pas de la provocation ou un caprice. C'est tout simplement impossible. Et pourtant, elle n'aime pas attirer l'attention sur elle et manger une pizza à Noël ou à un mariage alors qu'un bon repas est servi la distingue. Elle dit à plusieurs reprises qu'elle serait morte si elle n'avait pas pu bénéficier d'aménagements, car elle se serait laissé mourir de faim.

Au-delà de l'autonomie se dessine la quête d'identité. Pour Lamia, tant qu'elle ne pourra pas se nourrir ailleurs qu'à la maison, elle pourra rester dans le monde rassurant qu'elle s'est créé, un monde dans lequel elle endosse diverses identités imaginaires. Difficile de savoir qui «je» suis si «je» n'entre pas dans la norme, difficile de trouver des modèles dans le monde ordinaire quand «je» m'y sens exclue. Eloi ${ }^{15}, 13$ ans, un adolescent avec un TSA avec qui nous avons travaillé, avait évoqué cette difficulté: "Quand je vais à l'école, je dois être normal, alors je laisse mes avions à la maison. Quand je suis à la maison, je suis moi-même, je peux m'enfermer dans ma chambre et jouer avec mes avions.»

\section{Réunir - Manger, une histoire partagée}

Lors d'une rencontre, nous avons partagé une glace chez un glacier que Lamia aime beaucoup. Nous avons choisi chacune une glace qui nous plaisait. Lamia prend toujours la même glace, straciatella. J'ai pris une glace au sésame noir que je n'avais jamais goûtée.

Lors de la dégustation de la glace, nous avons chacune choisi notre parfum selon nos critères personnels. Nous avons ensuite parlé de nos choix et des raisons de ces choix. Ce fut une occasion de constater que les plaisirs de manger sont soumis à des motivations différentes, la certitude de retrouver un goût pour 
Lamia, la curiosité de découvrir un nouveau goût pour nous. Dans cet exemple, les protagonistes ont assumé leur subjectivité par le choix du parfum. Une situation dialogique a ainsi pu se créer. Il a été plus facile pour Lamia d'expliciter son choix et la chercheuse a pu mieux mesurer l'impact de pouvoir faire un choix sur le plaisir de manger, elle qui déteste l'arôme straciatella.

Pour notre cinquième rencontre, nous décidons de nous proposer l'une à

l'autre un aliment à déguster. Pour ce défi, je choisis deux aliments «bizarres», des chips à l'arôme improbable et un chocolat $100 \%$ cacao, car, comme le dit mon fils fatigué de ma cuisine trop originale à son goût, «tu n'arrives pas à choisir des trucs normaux!». Lamia n'est pas convaincue non plus par mes choix. Elle me dit qu'elle a choisi quelque chose de bon, «elle», et surtout quelque chose qu'elle a fait elle-même, des cookies et de la mousse au chocolat! Lors de la dégustation partagée, nous avons choisi, à notre habitude, des aliments spéciaux, des aliments qui demandaient à être expérimentés et non seulement à être mangés. Lamia est surprise par ce choix et elle explique le sien en contraste. De plus, elle a spécialement préparé les aliments en vue de notre rencontre. Elle a prévu les portions et les ustensiles pour deux. Le fait que la chercheuse ait assumé sa subjectivité, dans une logique dialogique, a aidé Lamia à expliquer ses motivations.

Lamia me raconte aussi ses expériences avec les bonbons Harry Potter. Ces bonbons lancent un défi aux enfants qui les consomment. Pour une même variante visuelle sont utilisés deux arômes, un habituel comme pêche, banane, myrtille, un inhabituel et repoussant comme vomi, œufs pourris, saleté... Lamia a relevé le défi plusieurs fois avec une amie jusqu’à ce que le jeu perde son intérêt.

Lamia peut aussi déguster les bonbons Harry Potter au risque de tomber sur un arôme repoussant et elle accepte de goûter les aliments que nous avons amenés et qui ne la tentent pas du tout. Ce sont des choix faits en toute connaissance de cause et librement consentis dans des contextes d'échange sociaux.

\section{Réjouir - Chronique d'un monde alimentaire intense et en perpétuel changement}

Lamia ne peut manger qu'un nombre restreint d'aliments. Ses arguments pour refuser un aliment sont souvent d'ordre sensoriel. Elle passe beaucoup de temps à détailler un aliment visuellement ou olfactivement. Elle distingue aisément de petites différences - dans le domaine visuel, trop de tomates sur le bord de la pizza, trop de petites graines; dans le domaine olfactif de petites différences entre le jambon de la marque $\mathrm{x}$ ou de la marque $\mathrm{y}$. Cette exploration sensorielle des aliments est caractéristique d'un traitement dit local des informations. Elle se fait souvent au détriment d'une reconnaissance globale des objets. Cette forme de traitement des informations est dominante chez les enfants avec un TSA contrairement aux enfants au DT qui privilégient un traitement global (Mottron, 2016; 
Mottron, Dawson, \& Soulières, 2009; Mottron, Dawson, Soulières, Hubert, $\&$ Burack, 2006). Dans une étude sur la façon dont 11 enfants (10.5 ans en moyenne) avec un TSA apprécient des images d'aliments (Luisier, 2017; Luisier et al., 2018), nous avons mis en évidence que ces enfants explorent tout aussi longuement des aliments simples (composés d'un seul aliment) que des aliments multiples (composés de plusieurs aliments) contrairement aux enfants au DT qui passent moins de temps avec les images d'aliments simples. Lamia semble consciente de cette façon de fonctionner car elle dit qu'elle ne doit pas passer trop de temps à «analyser», par exemple le cervelas qui fait partie des aliments qu'elle consomme régulièrement, sinon cela devient «dégueulasse». Avec certains aliments, comme la pizza, les brocolis ou le cervelas, il semblerait, du reste, qu'elle ait réussi à créer un concept suffisamment fort pour neutraliser le trop-plein d'évocations sensorielles.

Lamia m'a raconté que les enfants ne mangent pas les brocolis, mais qu'elle, elle les adorait. "Quand j'étais petite dans ma tête, vu que j'adore les dinosaures, j'étais un dinosaure qui mangeait les arbres et l'arbre, c'était le brocoli.»

Nous comprenons aussi que l'accumulation d'informations sensorielles amène Lamia à un moment ou à un autre vers des traces mémorisées désagréables, traces souvent sensorimotrices (Versace et al., 2014) qui la conduisent vers le refus de manger l'aliment. Lamia utilise peu de nuances pour porter son appréciation, elle aime ou elle déteste, c'est soit très bon, soit ignoble ou «dégueulasse». Les souvenirs ou l'expérience souvent d'ordre sensoriel qu'elle évoque pour refuser de manger un aliment sont violents - «ça me brûle l'estomac», "ça me défonce le nez», "ça va me cramer la langue». Elle est catégorique dans ses appréciations et cohérente dans son argumentation. "On ne peut pas m'avoir!»

Un monde perçu de façon si intense pourrait amener l'individu avec un TSA à développer, en guise de protection, des réactions de désengagement social et environnemental qui pourraient se traduire par l'adoption d'un répertoire restreint de routines sécurisantes qui se répètent (Markram, Rinaldi \& Markram, 2007). Pour Lamia, manger la cuisine de sa mère est la certitude de retrouver les mêmes perceptions sensorielles sans surprise, sans agression. Donner un ordre logique au repas, séparer les aliments pour éviter le mélange des goûts, donner une signification à l'aliment qui soit plus saillante que la perception sensorielle sont autant de stratégies qu'elle a réussi à mettre en place pour apprivoiser ce monde alimentaire trop agressif et trop changeant pour elle.

La question du sens donné à l'aliment est particulièrement intéressante. Manger est un acte particulier qui consiste non seulement à mettre en soi un aliment, mais qui conduit à utiliser des parties de cet aliment pour construire son propre corps. Au-delà de cette construction biologique, l'être humain ne peut s'empêcher de penser endosser, comme par magie, des propriétés plus symboliques des aliments qu'il ingère (Lahlou, 1996; Rozin, 1994). Cette dimension semble très importante chez Lamia. Des brocolis-arbres pour dinosaures aux 
cookies pour empereur nuisible empoisonnés par un apprenti sorcier, le monde alimentaire prend sens par l'histoire symbolique racontée.

Il est possible que pour Lamia, amener l'aliment dans le monde imaginaire qu'elle rejoint très souvent lui donne un sens, ce qui permet de créer une trace mnésique émotionnellement positive qui soit plus forte que l'expérience sensorielle négative, une façon de se distancier d'un monde sensoriel envahissant. Avec les aliments qu'elle a préparés pour la dégustation, Lamia mange et exprime son plaisir de manger. Elle a du plaisir à manger un aliment qui fait sens pour elle, un aliment doté d'une histoire, un aliment préparé avec soin pour notre rencontre, un aliment dont elle a suivi la recette à la lettre.

\section{En résumé}

À travers le recueil du récit de Lamia et son analyse, nous avons ainsi exploré le lien que Lamia a tissé avec le monde alimentaire. Nous comprenons qu'elle accepte de manger un aliment si:

- elle a la certitude de retrouver le "goût» attendu;

- l'aliment possède une signification suffisante à ses yeux;

- le repas respecte une logique qui fait partie de l'ordre des choses dans sa vision du monde.

Au-delà des facteurs sensoriels, Lamia dit ne pas aimer certains aliments, car elle s'est sentie forcée de les goûter ou de les manger. Elle se retrouve dans la situation de "dominée» qu'elle cherche à éviter à tout prix et que nous lions à sa quête d'autonomie. Cette question de l'autonomie, alimentaire ou autre, se retrouve comme un leitmotiv tout au long du récit. Lamia peine aujourd'hui à trouver sa place dans la société comme si elle ne se sentait pas entendue ou respectée. Ses difficultés alimentaires en sont un reflet.

Par la co-constuction du récit et de son interprétation, Lamia a pu écrire son histoire en s'appuyant sur ses interactions avec l'auteure. Par ce processus, elle a conscientisé son lien avec le monde alimentaire et elle a compris certaines de ses difficultés. Pour poursuivre l'accompagnement initié durant cette recherche, il s'agira de travailler la notion du sens que Lamia donne aux aliments et de trouver comment en donner dans des situations nouvelles de façon à mieux gérer les aspects sensoriels. D'un autre côté, il s'agira aussi de développer avec Lamia des ressources qui lui permettent avec le temps d'alléger son rapport à l'alimentation.

\section{Conclusion}

Cette recherche a investigué comment l'approche biographique permet de mieux comprendre le comportement alimentaire individuel de Lamia, une adolescente avec un TSA. Cette façon de procéder a permis (1) la création d'une relation entre Lamia et la chercheuse malgré des points de vue et l'appartenance à des «mondes» différents et (2) une co-construction de la compréhension du 
phénomène par l'investissement de cette relation comme espace de narration, de compréhension et de transformation des représentations.

D'une part, la reconfiguration du récit a permis la co-écriture de l'histoire (récit phénoménologique) alimentaire de Lamia. Cette étape précédant l'interprétation nous semble incontournable dans le cas du recueil du récit d'une personne qui dispose d'un mode de communication différent (Tager-Flusberg, Paul \& Lord, 2005) du nôtre afin d'assurer une bonne compréhension mutuelle. D'autre part, son interprétation a permis de mettre en évidence les enjeux que contient pour Lamia le monde alimentaire, les stratégies qu'elle a développées pour l'apprivoiser et d'ouvrir des pistes pour apaiser son rapport au monde alimentaire.

La démarche conduite avec Lamia a aussi eu des effets sur la chercheuse qui a recueilli le récit en toute sérendipité 16 et à la «seconde personne». Cette posture a demandé de se remettre en question, d'assumer ses manques et ses doutes sans honte ${ }^{17}$, de lâcher prise sur ses convictions personnelles ou sur les normes sociales, d'être consciente à la fois de savoir, expertise essentielle, et que ce savoir est questionnable, d'adopter à chaque rencontre un regard neuf comme s'il ne connaissait rien au monde de l'autre, d'explorer des domaines inédits, et surtout d'accueillir sa propre surprenance ${ }^{18}$ pour se laisser transformer. "Construisant du sens par rapport à nous-mêmes, construisant du sens par rapport à l'autre, nous élargissons notre capacité herméneutique, nous complexifions notre modèle d'intelligibilité (Delory-Momberger, 2002, p. 283)». Au-delà, quand la chercheuse porte un regard sur son propre vécu («première personne»19) durant les différentes phases du recueil et de l'analyse, elle peut mieux identifier la place de sa subjectivité dans ses choix théoriques.

L'histoire de Lamia ne peut bien sûr en aucun cas être généralisée à toutes les personnes avec un TSA. Bien au contraire, elle montre combien la procédure de construction de sens gagne à être abordée de façon individuelle à travers la subjectivité de la personne. Ainsi, le recueil du récit, la co-contruction du récit phénoménologique et de son interprétation ouvrent des perspectives intéressantes pour l'accompagnement de la construction alimentaire, que ce soit par des professionnels de la santé ou des enseignants en éducation nutritionnelle non seulement chez les personnes avec un TSA mais auprès de toutes celles qui rencontrent des difficultés avec leur alimentation.

\section{Notes}

1 Lamia, prénom d'emprunt choisi par la narratrice elle-même.

2 Pour des raisons de simplification du texte, nous utiliserons indistinctement la dénomination «enfant» ou «adolescent» pour «enfant et adolescent».

3 Même si aujourd'hui les politiques publiques devraient tendre vers des aménagements, produits, équipements ou services basés sur une conception universelle accessible à tous, quels que soient l'âge, le sexe, le handicap ou la situation (voir Ginnerup, 2009), dans la réalité, les prestations publiques ordinaires proposées, par exemple dans le domaine de l'éducation ou de la promotion de la santé, ne conviennent pas toujours à tous. C'est cette compréhension du mot ordinaire que nous utilisons dans le cadre de cet article. 
Il n'y a pas de consensus sur la définition des problèmes ou difficultés alimentaires dans la littérature scientifique (pour une revue voir Nadon et al., 2013), ce qui peut expliquer la largeur des fourchettes d'occurrences.

5 Il n'existe pas de définition consensuelle de la sélectivité alimentaire si ce n'est la consommation d'un nombre restreint d'aliments (Bandini et al., 2010).

6 «En un mot, l'émancipation est le mouvement par lequel l'homme ou la femme prenant conscience de ce qui empêche la volonté d'accéder à son propre désir et de le mettre en œuvre, initie une dynamique de production et de réalisation du sens dans le cours de sa propre existence.» (Brun, 2001, p. 158)

7 Cela "permet à des individus qui se vivaient jusque-là entièrement soumis à une histoiredestin, de passer du statut d'objet à celui de sujet de leur histoire, et d'acquérir ainsi la conviction qu'ils peuvent changer quelque chose à leur vie à venir.» (Lainé, 2004, p. 168).

8 Ces dégustations sont décrites dans la partie résultats. Nous nous sommes très librement inspirées des approches en ethnographie sensorielle (Pink, 2015).

9 Katja Vanini de Carlo (Vanini De Carlo, 2014), suite aux travaux de Bruner (Bruner, 1991), pose comme dimensions centrales de cette modélisation narrative:

- la reconstruction des différents temps (du vécu, de la narration, de l'interprétation, ...);

- la mise en évidence d'évènements particuliers;

- la narration autant «de l'advenu que de la raison pour laquelle il importe de le raconter»;

- la distinction «entre l'intention et la raison d'agir».

10 En référence au cadre théorique des histoires de vie.

11 Dans le cadre de cet article, l'auteure utilisera le «nous» académique quand il s'agit d'interpréter les résultats et le «je» quand elle relate son implication dans le dialogue avec les différents sujets d'étude.

12 Le récit phénoménologique complet peut être obtenu en s'adressant à l'auteure.

13 Nous nous sommes permis d'opérationnaliser la notion de "pics de ralentissement» sans passer par le calcul effectif du temps, comme proposé par Vanini de Carlo (2014b). Nous nous sommes basée à la fois sur la perception du temps que nous avons ressenti durant l'expérience (comme une psycho-phénoménologie des micro-moments (de Champlain, 2011)) et sur la récurrence du phénomène.

14 Le terme "goût» est compris dans son acceptation large, comme perçu par tous les sens.

15 Prénom d'emprunt

16 "[...] une démarche d'enquête dont le cheminement même n'est pas prédéfini, et peut conduire à un changement de paradigme» (Catellin \& Loty, 2013).

17 Vincent de Gaulejac postule que dans la relation d'aide comme dans la recherche scientifique, l'accompagnant ou le chercheur doit faire face à ses propres hontes pour pouvoir sortir du sentiment d'être dans une position dominante face à la personne qui est accompagnée ou étudiée. «C'est surtout de sa capacité à être en relation avec sa propre honte que dépend la possibilité d'être en relation avec ceux pour qui «elle colle à la peau» (De Gaulejac, 1996, pp. 295-296).

18 Concept «inventé» par Jean-François Malherbe qu'il définit comme la disposition des individus - et de l'Univers aussi - à produire des surprises, c'est-à-dire à être différent de ce que j'attends, comme une part de moi-même qui me surpasse.

19 Pour une réflexion plus approfondie sur la recherche en "première, seconde et troisième personne», voir Vermersch, 2010. 


\section{Bibliographie}

Agence nationale de sécurité sanitaire de l'alimentation, de l'environnement et du travail [ANSES]. (2010). Évaluation des risques liés aux pratiques alimentaires d'amaigrissement (Rapport d'expertise collective). Maisons-Alfort Cedex. Consulté dans https://www.anses. $\mathrm{fr} / \mathrm{fr} /$ system/files/NUT2009sa0099Ra.pdf

APA. (2015). DSM-5 - Manuel diagnostique et statistique des troubles mentaux - traduction française ( 5 éd.). Completer avec Lieu: maison d'édition.

Bandini, L. G., Anderson, S. E., Curtin, C., Cermak, S., Evans, E. W., Scampini, R., ... préciser tous les auteurs!! Must, A. (2010). Food selectivity in children with autism spectrum disorders and typically developing children. The Journal of pediatrics, 157, (2), 259-64. http://doi.org/10.1016/j.jpeds.2010.02.013

Brun, P. (2001). S'émanciper de la misère: le parcours de vie d'une femme québécoise. Carriérologie, $1,157-171$.

Bruner, J. (1991). The Narrative Construction of Reality. Critical Inquiry, 18, (1), 1-21.

Catellin, S. \& Loty, L. (2013). Sérendipité et indisciplinarité. Hermès, La Revue, 3, (67), 32-40.

Cermak, S. A., Curtin, C. \& Bandini, L. G. (2010). Food selectivity and sensory sensitivity in children with autism spectrum disorders. Journal of the American Dietetic Association, 110, (2), 238-246. http://doi.org/10.1016/j.jada.2009.10.032

Chiva, M. (1979). Comment la personne se construit en mangeant. Communications, 31(1), 107-118. https://doi.org/10.3406/comm.1979.1472

Chiva, M. (1985). Le Doux et l'Amer: Sensation gustative, Emotion et Communication chez le jeune enfant. Paris, France: Presses Universitaires de France - PUF.

de Champlain, Y. (2011). Articuler son histoire de moment en moment: Psycho-phénoménologie des micro-moments. In P. Galvani, D. Nolin, Y. de Champlain \& G. Dubé (Éd.), Moments de formation et mise en sens de soi (pp. 97-110). Paris, France: L'Harmattan.

De Gaulejac, V. (2008). Les sources de la honte. Paris, France: Desclée de Brouwer.

de Suremain, C.-H. \& Razy, E. (2012). L'alimentation de l'enfant. In J.-P. Poulain (Éd.), Dictionnaire des cultures alimentaires (pp. 451-458). Paris, France: PUF.

Delory-Momberger, C. (2002). Les histoires de vie, une herméneutique auto-éducative dans l'interculturel. In Souci et soin de soi (pp. 261-284). Paris, France: L'Harmattan.

Donnellan, A. M., Hill, D. a. \& Leary, M. R. (2013). Rethinking autism: implications of sensory and movement differences for understanding and support. Frontiers in integrative neuroscience, 6, (124), 1-11. http://doi.org/10.3389/fnint.2012.00124

Etiévant, P., Bellisle, F., Dallongeville, J., Etilé, F., Guichard, E., Padilla, M. \& Romon-Rousseaux, M. (2010). Les comportements alimentaires. Quels en sont les déterminants? Quelles actions pour quels effets? Expertise scientifique collective, rapport. France: INRA. Consulté dans http://inra.dam.front.pad.brainsonic.com/ressources/afile/223304-f53c6-resource-expertise-comportements-alimentaires-rapport-complet.html

Fischler, C. (2012). Commensalité. In J.-P. Poulain (Ed.), Dictionnaire des cultures alimentaires. Paris, France: Presses Universitaires de France - PUF.

Fodstad, J. C. \& Matson, J. L. (2008). A comparison of feeding and mealtime problems in adults with intellectual disabilities with and without autism. Journal of Developmental and Physical Disabilities, 20, (6), 541-550. http://doi.org/10.1007/s10882-008-9116-6

Ginnerup, S. (2009). Assurer la pleine participation grâce à la conception universelle. Retrieved from http://reglementationsaccessibilite.blogs.apf.asso.fr/media/00/02/510804250.2.pdf

Herndon, A. C., DiGuiseppi, C., Johnson, S. L., Leiferman, J. \& Reynolds, A. (2009). Does nutritional intake differ between children with autism spectrum disorders and children with typical development? Journal of autism and developmental disorders, 39, (2), 212-22. http://doi.org/10.1007/s10803-008-0606-2

Johnson, C. R., Foldes, E., Demand, A. \& Brooks, M. M. (2015). Behavioral parent training to address feeding problems in children with autism spectrum disorder: A pilot trial. 
Journal of Developmental and Physical Disabilities, 27, 591-607. http://doi.org/10.1007/ s10882-015-9437-1

Lahlou, S. (1996). Cuisinons la représentation sociale. Pensée magique et alimentation aujourd'hui. Les Cahiers de l'OCHA, 5, 10-17.

Lainé, A. (2004). Faire de sa vie une histoire: Théories et pratiques de l'histoire de vie en formation. Paris, France: Desclée de Brouwer.

Lecerf, J.-M. (2013). A chacun son vrai poids. Paris, France: Odile Jacob. Consulté dans http:// www.amazon.fr/chacun-son-vrai-poids-ebook/dp/B00BQ2S7HY

Ledford, J. R. \& Gast, D. L. (2006). Feeding Problems in Children With Autism Spectrum Disorders: A Review. Focus on Autism and Other Developmental Disabilities, 21, (3), 153-166. http://doi.org/10.1177/10883576060210030401

Luisier, A.-C. (2017). Perception visuelle et olfactive chez les enfants avec un trouble du spectre de l'autisme : implications sur l'acceptation des aliments. Thèse de doctorat, école doctorale Neurosciences et Cognition, Université de Lyon 1 et Université de Fribourg.

Luisier, A.-C., Petitpierre, G., Bérod, A. C., Richoz, A.-R., Lao, J., Caldara, R. \& Bensafi, M. (2018). Visual and hedonic perception of food stimuli in children with autism spectrum disorders and their relationship to food neophobia.

Luisier, A.-C., Petitpierre, G., Ferdenzi, C., Bérod, A. C., Giboreau, A., Rouby, C. \& Bensafi, M. (2015). Odor Perception in Children with Autism Spectrum Disorder and its Relationship to Food Neophobia. Frontiers in Psychology, 6, (1830), 1-10. http://doi. org/10.3389/fpsyg.2015.01830

Markram, H., Rinaldi, T. \& Markram, K. (2007). The Intense World Syndrome - an alternative hypothesis for autism. Frontiers in neuroscience, 1, (1), 77-96.

Marshall, J., Hill, R. J., Ziviani, J. \& Dodrill, P. (2013). Features of feeding difficulty in children with Autism Spectrum Disorder. International Journal of Speech-Language Pathology, 16, (2), 151-158. http://doi.org/10.3109/17549507.2013.808700

Matson, J. L., Fodstad, J. C. \& Dempsey, T. (2009). The relationship of children's feeding problems to core symptoms of autism and PDD-NOS. Research in Autism Spectrum Disorders, 3, (3), 759-766. http://doi.org/10.1016/j.rasd.2009.02.005

Mottron, L. (2016). L'intervention précoce pour enfants autistes Nouveaux principes pour soutenir une autre intelligence. Bruxelles: Mardaga.

Mottron, L., Dawson, M. \& Soulières, I. (2009). Enhanced perception in savant syndrome: patterns, structure and creativity. Philosophical transactions of the Royal Society of London. Series B, Biological sciences, 364, (1522), 1385-91. http://doi.org/10.1098/rstb.2008.0333

Mottron, L., Dawson, M., Soulières, I., Hubert, B. \& Burack, J. (2006). Le surfonctionnement perceptuel dans l'autisme. Une mise à jour, et huit principes sur la perception autistique. Revue de Neuropsychologie, 16, (3), 251-297.

Nadon, G., Ehremann Feldman, D. \& Gisel, E. (2008). Revue des méthodes utilisées pour évaluer l'alimentation des enfants présentant un trouble envahissant du développement. Archives de pédiatrie, 15, 1332-1348.

Nadon, G., Feldman, D. E., Dunn, W. \& Gisel, E. (2011). Mealtime problems in children with autism spectrum disorder and their typically developing siblings: a comparison study. Autism : the international journal of research and practice, 15, (1), 98-113. http:// doi.org/10.1177/1362361309348943

Nadon, G., Feldman, D. \& Gisel, E. (2013). Feeding issues associated with the Autism Spectrum Disorders. In M. Fitzgerald (Éd.), Recent advances in Autism Spectrum Disorders Volume I (pp. 597-630). Rijeka: InTech. http://doi.org/http://dx.doi.org/10.5772/53644

Paillé, P. \& Mucchielli, A. (2016). L'analyse qualitative en sciences humaines et sociales (4e éd.). Malakoff, France: Armand Colin.

Pineau, G. \& Legrand, J.-L. (2013). Les histoires de vie (5e éd.). Paris, France: Presses Universitaires de France - PUF.

Pink, S. (2015). Doing sensory ethnography (2nd éd.). London: SAGE Publications. 
Rommetveit, R. (2003). On the role of " a psychology of the second person " in studies of meaning, language, and mind. Mind, Culture and Activity, 10, (3), 205-2018. http://doi. org/10.1207/s15327884mca 1003

Rozin, P. (1994). La magie sympathique. In C. Fischler (Éd.), Manger magique. Aliments sorciers, croyances comestibles. (Vol. 149, pp. 22-37). Paris: Autrement - collection Mutations.

Schaaf, R. C., Toth-Cohen, S., Johnson, S. L., Outten, G. \& Benevides, T. W. (2011). The everyday routines of families of children with autism: Examining the impact of sensory processing difficulties on the family. Autism: the international journal of research and practice, 15, (3), 373-389. http://doi.org/10.1177/1362361310386505

Sharp, W. G., Jaquess, D. L. \& Lukens, C. T. (2013). Multi-method assessment of feeding problems among children with autism spectrum disorders. Research in Autism Spectrum Disorders, 7, (1), 56-65. http://doi.org/10.1016/j.rasd.2012.07.001

Souriau, J. (2013). Comprendre et communiquer avec ceux qui ne parlent pas. Vie sociale, 3, (3), 93-116. http://doi.org/10.3917/vsoc.133.0093

Tager-Flusberg, H., Paul, R. \& Lord, C. (2005). Language and communication in autism. In F. R. Volkmar, R. Paul, A. Klin, \& D. Cohen (Éd.), Handbook of Autism and Pervasive Developmental Disorders (Vol. 1, pp. 335-364). New Jersey, NJ: John Wiley \& Sons, Inc. http://doi.org/10.1002/9780470939345.ch12

Vanini De Carlo, K. (2014). Se dire e(s)t devenir - La recherche biographique comme choix épistémologique. ¿ Interrogations ?, 17, 1-14. Consulté dans http://www.revue-interrogations.org/Se-dire-e-s-t-devenir-La-recherche, 401

Vermersch, P. (2010). Les points de vue en première, seconde et troisième personne dans les trois étapes d'une recherche : conception, réalisation, analyse. Expliciter, 85, 19-32.

Versace, R., Vallet, G. T., Riou, B., Lesourd, M., Labeye, É. \& Brunel, L. (2014). Act-In: An integrated view of memory mechanisms. Journal of Cognitive Psychology, 26, (3), 280-306. http://doi.org/10.1080/20445911.2014.892113

Wethington, E. (2005). An overview of the life course perspective: Implications for health and nutrition. Journal of nutrition education and behavior, 37, (3), 115-120.

Zimmer, M. H., Hart, L. C., Manning-Courtney, P., Murray, D. S., Bing, N. M. \& Summer, S. (2012). Food variety as a predictor of nutritional status among children with autism. Journal of autism and developmental disorders, 42, (4), 549-556. http://doi.org/10.1007/ s10803-011-1268-z

Mots-clés: Perception sensorielle, trouble du spectre de l'autisme, éducation nutritionnelle, histoires de vie

\section{Biografische Begleitung und Aufbau des Essverhaltens bei Kindern mit einer Autismus-Spektrum-Störung}

\section{Zusammenfassung}

Bei Kindern und Jugendlichen mit Autismus-Spektrum-Störungen (ASS) treten oft Ernährungsprobleme auf, insbesondere ein hoch selektives Essverhalten, das ihr Leben und dasjenige ihrer Angehörigen erschwert. Diese qualitative Forschung verwendet für die Fallstudie einer Jugendlichen mit einer ASS und einem hochselektivem Essverhalten einen biografischen Ansatz. Nach einer kurzen Darstellung der ASS sollen zunächst die dem Essensakt zugrundeliegenden Problematiken beschrieben werden. Anschliessend wird die Verbindung, die die 
Jugendliche zur Ernährungswelt geschaffen hat, im Dialog mit der Neurowissenschaft interpretiert und damit die Bedeutung des Methodenmix (qualitative und quantitative Methoden) in der Ernährungsbegleitung aufgezeigt.

Schlagworte: Sensorische Wahrnehmung, Autismus-Spektrum-Störungen, Ernährungserziehung, biographische Ansatz, Neurowissenschaft

\section{Accompagnamento biografico e costruzione del comportamento alimentare nei bambini con disturbo dello spettro autistico}

\section{Riassunto}

I bambini e gli adolescenti con disturbo dello spettro autistico (DSA) sono frequentemente soggetti a problemi alimentari, soprattutto correlati a un'elevata selettività che rende complicata la loro vita e quella dei loro cari. Questa ricerca di matrice qualitativa si avvale di un approccio biografico per svolgere uno studio di caso su un'adolescente con DSA che presenta una forte selettività alimentare. In una prima fase, dopo una breve presentazione dei DSA, vengono descritti $\mathrm{i}$ fattori strategici alla base dell'atto alimentare. A seguire vengono proposti alcuni elementi di comprensione del legame che l'adolescente ha instaurato con la sfera alimentare sulla base della sua storia personale.

Parole chiave: Percezione sensoriale, disturbi dello spettro autistico, educazione alimentare, biografie

\section{Biographical counselling and construction of eating behaviour in children with Autism Spectrum Disorder}

\section{Summary}

Children and adults with an autistic spectrum disorder (ASD) frequently experience dietary challenges, most notably in the highly selective eating habits which complicate their lives and those of the people who care for them. The qualitative research conducted here uses a biographical approach to carry out a case study of an adolescent girl with an ASD who demonstrates a high degree of selective eating. Initially, a brief presentation of ASD is made, and the main issues which underlie the act of eating are described. The connection the girl has forged with the world of food is then viewed through an approach that draws on neuroscience to demonstrate the advantages of using mixed methods (qualitative and quantitative) when providing nutritional support.

Keywords: Sensory perception, autistic spectrum disorder, nutrition education, life history, neuroscience 